\title{
超晶格 $\mathrm{YBa}_{2} \mathrm{Cu}_{3} \mathrm{O}_{7} / \mathrm{PrBa}_{2} \mathrm{Cu}_{3} \mathrm{O}_{7}$ 的 $\mathbf{X}$ 射线衍射研究
}

\author{
李可斌 \\ (中国科学院固体物理研究所, 合肥 230031) \\ 姚伟国 \\ (苏州大学物理系, 苏州 215006) \\ 戚震中 \\ (上海交通大学信息存储中心, 上海 200030)
}

\section{关键词超晶格 $\mathrm{YBa}_{2} \mathrm{Cu}_{3} \mathrm{O}_{7} / \mathrm{PrBa}_{2} \mathrm{Cu}_{3} \mathrm{O}, \quad \mathrm{X}$ 射线衍射}

随着薄膜制备技术的发展, 高质量的高温超导超晶格 $\mathrm{YBa}_{2} \mathrm{Cu}_{3} \mathrm{O}_{7} / \mathrm{PrBa}_{2} \mathrm{Cu}_{3} \mathrm{O}_{7}$ 样品的获 得 ${ }^{[1,2]}$ 为实验研究高温超导有关物性方面提供了其它试样所无法替代的可能性, 其中一个非常 有意义的特性是当一比较薄的 YBCO 超导层被 PrBCO 层隔开时, 其 $T_{\mathrm{c}}$ 将随着非超导层 $\operatorname{PrBCO}$ 层的厚度增加而下降 ${ }^{[1,2]}$, 据此在理论上也相应地提出了各种解释模型, 诸如自旋极化模型 ${ }^{[3]}$ 、

Kosterlitz-Thouless 相变模型 ${ }^{[4]}$ 及最近临效应模型 ${ }^{[\mathrm{g}}$ 等. 但所有这些理论模型都假设超晶格间 是一种完整的多层结构, 忽略了层间可能存在的无序性及界面的存在, 包括界面原子间的扩 散、层错及应力等对 $T_{\mathrm{c}}$ 的影响. 本文将比较详细地报道系列超晶格 $(\mathrm{YBCO})_{n} /(\mathrm{PrBCO})_{m}$ 薄膜 样品的 X 射线衍射研究结果 (调制比分别为 $1: 1,1: 3,1: 5,1: 8,2: 15$ ), 并比较采用 SUPREX 程序所得到的模拟计算 XRD 谱图, 对样品中可能存在的上面所提到的缺陷作了定量的分析.

\section{1 样品的制备}

$(\mathrm{YBCO})_{n} /(\mathrm{PrBCO})_{m}$ 超晶格薄膜采用直流贼射法沉积在 $\mathrm{SrTiO}_{3}(100)$ 衬底上, 沉积时基片温 度为 $810^{\circ} \mathrm{C}$ 左右, 贼射是在纯氧气氛中进行的, 氧压通常为 $3 \mathrm{mbar}$, 溅射用的 $\mathrm{YBCO}$ 靶和 $\mathrm{PrBCO}$ 靶都是采用化学配比为 $1: 2: 3$ 相的平面靶, 薄膜的生长率近似为 $0.03 \sim 0.04 \mathrm{~nm} / \mathrm{s}$, 超晶格的调 制比是由控制基片在各浌射靶上沉积时间来决定, 这种沉积时间通过计算机对步进马达自动 控制而得到, 浌射时旋转基片座往复于 $Y$ 靶和 Pr 靶之间, 直至最后膜厚为 $200 \mathrm{~nm}$ 左右, 详细 制备方法可参见文献[6].

\section{$2 \mathrm{X}$ 射线衍射结果及与由 SUPREX 程序模拟计算结果的比较}

图 1-1 给出了调制比为 $1: 1$ ( 调制波长为 $\Lambda=1 C_{\mathrm{Y}}+1 C_{\mathrm{Pr}} \approx 2 C_{\mathrm{Y}}=2.4 \mathrm{~nm}$ ) 的超晶格薄膜( $\mathrm{YP} 147$ ) 的 $\mathrm{X}$ 射线衍射结果, 两相邻主峰 (001) 之间有一相当高强度的伴峰, 这是由多层膜结构衍射的 结果, 而且对主峰 (005) 峰的摇摆曲线的测量表明其半高全峰宽为 $0.3^{\circ}$ 左右, 表明此膜是一个

1994-11-23 收稿, 1995-03-20 收修改稿 


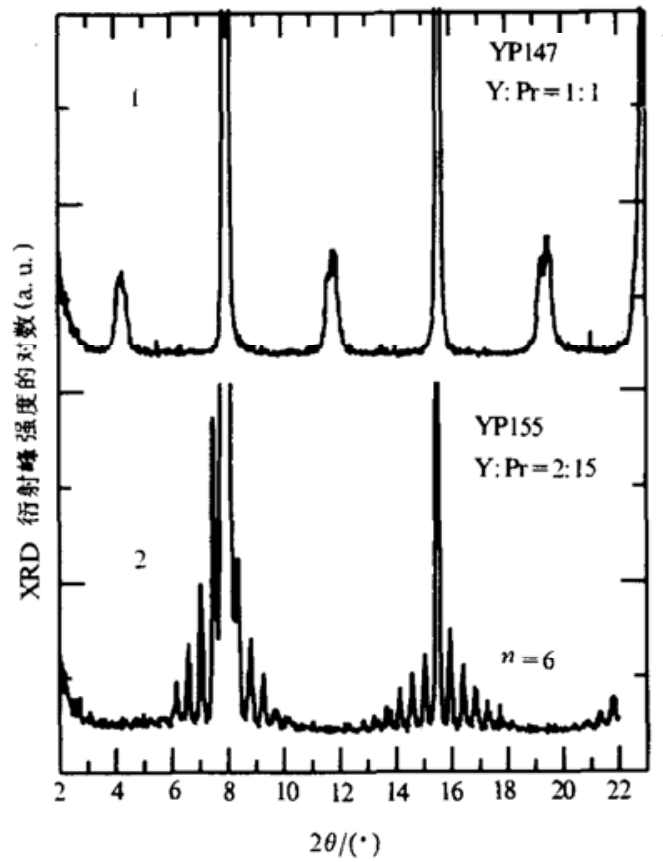

图 $1 X$ 射线衍射结果

1 为样品 YP147( 调制比为 1:1), 2 为调制比为 $2: 15$ 的 样品 YP155 的结果, 可以明显地观察到第 6 级伴峰

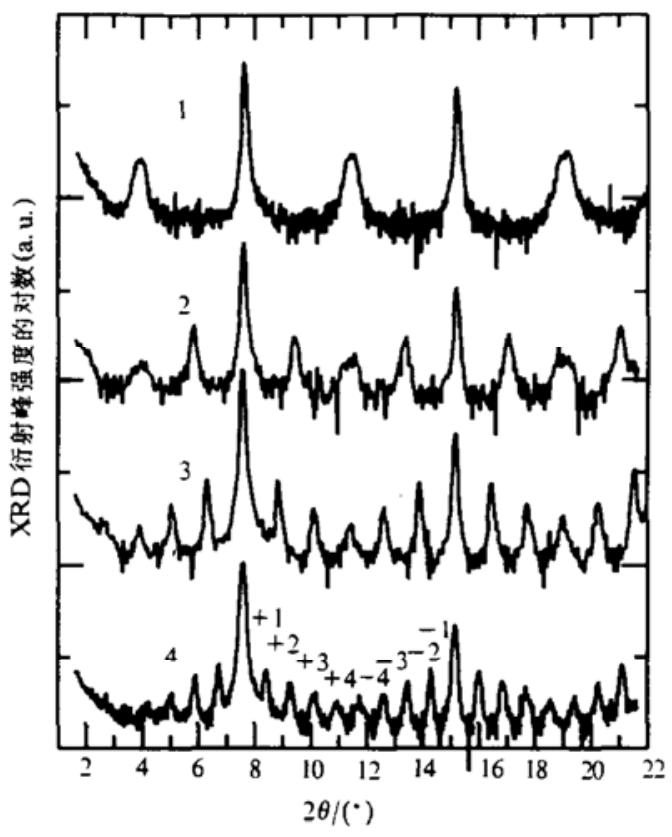

图 2 儿个样品的 $\mathrm{X}$ 射线衍射结果 纵轴为衍射强度取对数后的结果, 图中的标岇分别对应 样品, 1 为 YP147(1:1), 2 为 YP148(1:3), 3 为 YP149 (1:5), 4 为 YP164(1:8)

高质量的 $c$ 轴外延取向薄膜. 图 1-2 所给出的是调制比为 2:15 的另一超晶格试样 YPI55 的 X 射线衍射结果, 两相邻主峰间的伴峰结构可以清楚地观察到第 6 级. 同样地我们测量了调制 比分别为 $1: 3,1: 5$ 和 $1: 8$ 的其它几个样品的 $X$ 射线衍射结果. 为了比较起见, 我们将它们的 XRD 谱在 $2 \theta$ 从 $2^{\circ}$ 到 $23^{\circ}$ 的范围内一起画在图 2 上, 其中的纵坐标是对衍射强度取对数后得 到的. 由此我们可以看出, 对于单原胞 YBCO 层厚的超晶格样品的两主峰间的伴峰个数等于 PrBCO 层的原胞数, 如样品 YP164(1:8) 两主峰 (001) 和 (002) 间有 8 个伴峰, 它们分别 是: $(001)$ 主峰的 “ +1 ” “ +2 ” “ +3 ” “ +4 ”级伴峰及 $(002)$ 主峰的 “ -1 ” “- -2 ” -3 ” “ -4 ”级伴峰, 这 种多层膜的伴峰结构可以作如下解释:

考虑 $(\mathrm{YBCO})_{n} /(\mathrm{PrBCO})_{m}$ 超晶格实际结构简图, 其调制波长为 $A=C 1 n+C 2 m$, 重复周期为 $M$ 次, 若只考虑一个单元中的二层系统的 $\mathrm{X}$ 射线衍射情形, 其结构因子应为

$$
S^{\prime}=\sum_{j=1}^{n_{1}} S_{1} \mathrm{e}^{-i q C_{1} J}+\mathrm{e}^{-i q C_{1} n_{1}} \sum_{j=1}^{n_{2}} S_{2} \mathrm{e}^{-i q C_{2} j},
$$

其中 $S_{1}, S_{2}$ 分别为各单层中的原子结构因子. 那么对于这样一种多层超晶格系统, 其总的结 构因子为

则衍射强度为

$$
S_{S L}=\sum_{K=1}^{M} S^{\prime} \mathrm{e}^{-i q \Lambda K}
$$

$$
I \rightsquigarrow\left[S_{S L}\right]^{2}=\left[\sin ^{2}\left(\frac{M}{2} q \Lambda\right) / \sin ^{2}\left(\frac{1}{2} q \Lambda\right)\right]\left|S_{1} \frac{1-\mathrm{e}^{-i q C_{1} n_{1}}}{1-\mathrm{e}^{-i q C_{1}}}+S_{2} \mathrm{e}^{-i q c_{1} n_{1}} \frac{1-\mathrm{e}^{-i q C_{2} n_{2}}}{1-\mathrm{e}^{-i q C_{2}}}\right|^{2} .
$$


由此可得 $q \Lambda=2 \pi p$, 也即 $p \lambda=2 \Lambda \sin (\theta)=2(n+m) C \sin (\theta)$ 从而有 $p=1(n+m)$. 这里假设 了 $C_{1}=C_{2}=C$, 因此在两主峰间就增加了 $n+m-1$ 个超晶格伴峰, 这是完全由多层膜的结构 所产生的一种衍射峰, 对于调制比为 $n: m=1: 8$ 的情形, 介于 (001) 和 (002) 峰间就存在 8 个伴 峰, 这与实验观察到的结果是一致的. 另外, 我们由衍射强度公式还可以看出, 如果上式中的 绝对值里的值为零, 则此处的 $I=0$, 即出现所谓的衍射峰的消失, 也就是说不是所有衍射峰都 能出现的, 在此就不作详细的说明了.

由于 $X$ 射线衍射实验得到的只是一种平均效果, 无法定量地反映样品内部的精细结构, 也就是说光从实验结果来看无法定量地给出层间是否存在原子间的扩散 (特别是 $Y$ 原子和 $P r$ 原子间的扩散), 而且也不知道层与层间的界面粗粘度到底有多大? 为进一步研究这些问 题, 我们借助于 Vanderstraeten 及 Schuller 等沉发展起来的 SUPREX 计算程序, 在计算模型中 引人台阶无序、缺陷、原子间的扩散及层厚的波动等因素对各个样品的 XRD 结果作了理论计 算模拟, 然后比较实验测得的谱图, 从而对我所制得的超晶格薄膜中的内部精细结构作了定 量的分析.

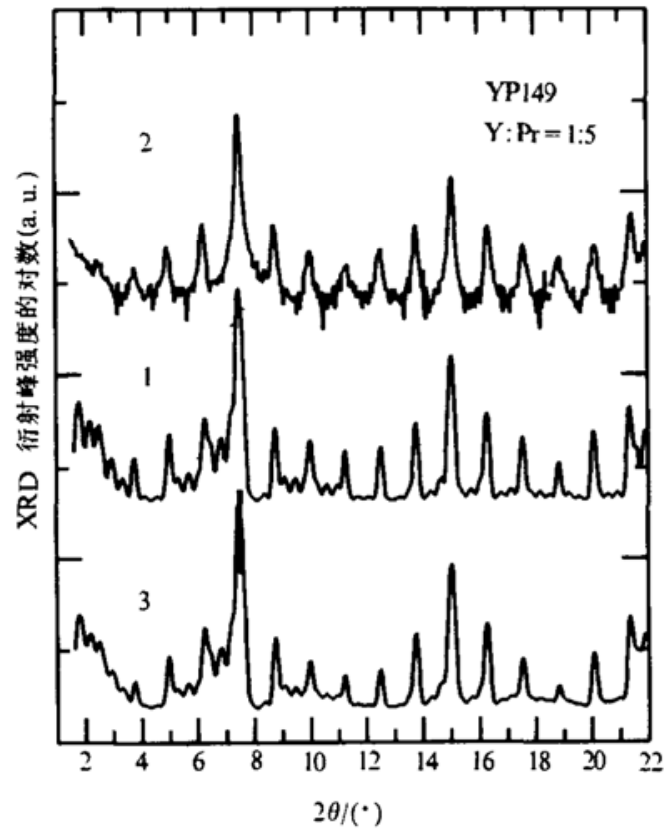

图 3 样品 YP149(1:5)X 射线衍射的实验结果 (2) 和理论模拟计算结果的比较

1 为理想 $1: 5$ 结构的模拟结果, 3 为考虑各种影响后的实 际结构的模拟计算后的结果

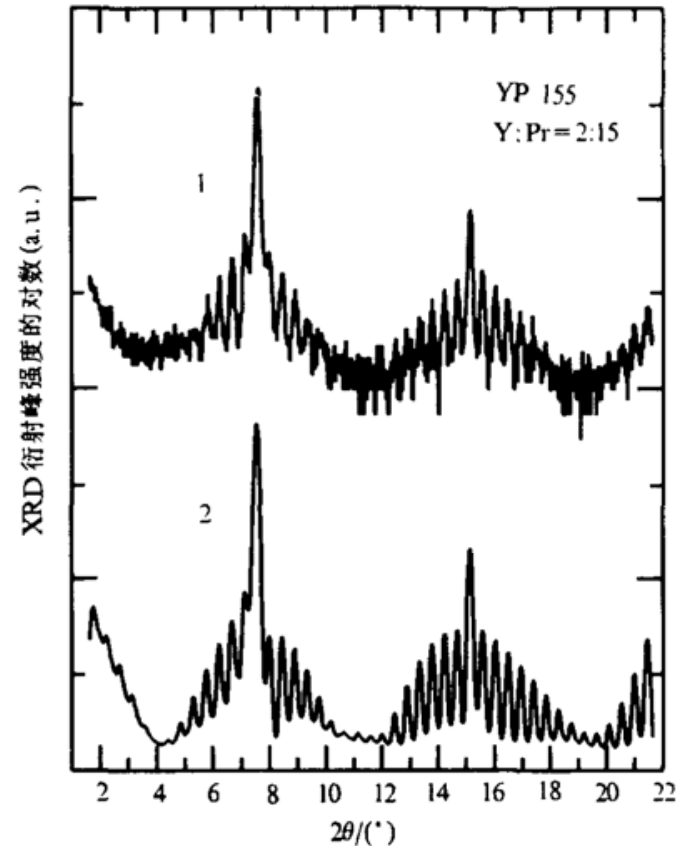

图 4 样品 YP155(2:15)X 射线衍射的实验结果 (1) 与考虑各种影响后的更实际结构的理论模拟计算. 结果 (2)

比较发现两者在峰形及伴峰相对强度上都较好的符合

图 4 给出了调制比为 1:5 超晶格薄膜 YP149 的 X 射线衍射的实验结果和理论模拟结果 的比较, 其中谱 1 为理想超晶格 1:5 结构的模拟谱图, 比较实验测得的谱 2 发现尽管两者定性 上符合得很好, 但非常明显, 在具体细节上仍然差别很大, 即在峰的线宽及伴峰对主峰的相对 强度上存在着分歧, 实验上观察到的伴峰要比模拟计算的要展宽些, 而且相对强度要低些. 考虑到界面原子间的扩散可能会影响到衍射强度, 以及界面上的缺陷、层错等无序性及层厚 
的微小波动会影响到衍射峰的强度又会影响到线宽的事实, 适当引人这两种效应后, 对谱 1 作 了修正后如谱 3 所示. 其中在计算谱 3 时, 假设界面原子的粗糙度为 $\sigma_{d}=0.003 \mathrm{~nm}$, 层厚波动 为 $\sigma_{n} / n=5 \%$ 及 $\mathrm{Y}$ 原子和 $\mathrm{Pr}$ 原子间的扩散量为 $\operatorname{diff}=10 \%$, 其中的层厚波动量可以理解为存 在着 $5 \%$ 的可能性使得在界面处某些地方可能存在着 0 个原胞层厚的 YBCO 原胞层, 而在另 外的地方存在着 2 个原胞层厚的 YBCO 层. 显然修正后的谱 3 更符实验结果, 这表明在 1:5 的样品中确实存在着前面所提的结构上的不完整性。在图 5 中类似地给出了调制比为 $2: 15$

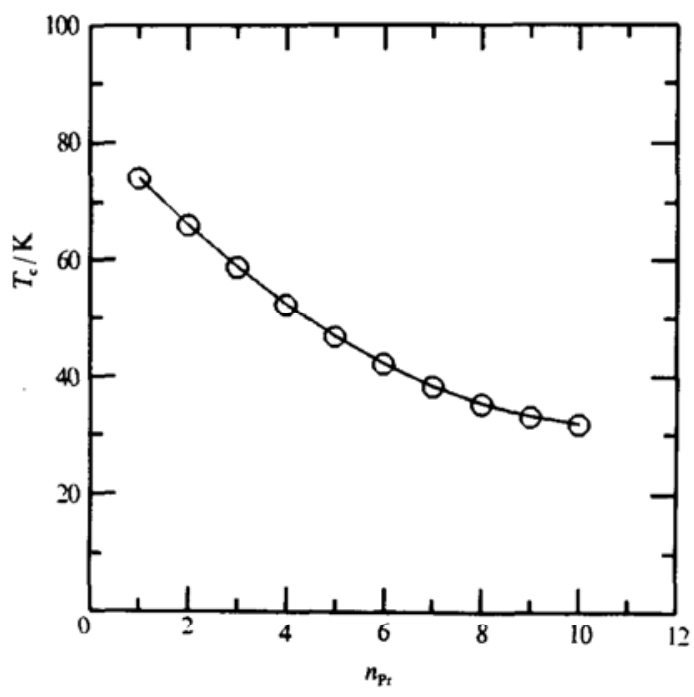

图 5 单个 $\mathrm{YBCO}$ 原胞层厚的 $\mathrm{YBCO}, \mathrm{PrBCO}$ 超导 超晶格样品的 $T_{\mathrm{c}}$ 与 $\mathrm{PrBCO}$ 层原胞数 $n_{\mathrm{Pr}}$ 间的关系, 随 着原胞数 $n_{\mathrm{Pr}}$ 的增加 $T_{\mathrm{c}}$ 下降 的超晶格样品 YP155 的 XRD 研究结果. 1 为实验测量谱, 2 为修正后的模拟谱, 在谱 3 的计算中考虑了 $\sigma_{d}=0.003 \mathrm{~nm}, \operatorname{diff}=15 \%$, $\sigma_{n} / n=5 \%$, 两者无论在形状和峰的相对强 度上都符合得很好. 类似地我们对其它几 个样品也作了同样的研究, 在此不作一一描 述了, 只是在表 1 中罗列了计算时所用的 YBCO 的面间距的值及相应的修正参数.

由表我们可以看出, 界面粗䊁度 $\sigma d$ 最 大为 $0.01 \mathrm{~nm}$, 最小为 $0.002 \mathrm{~nm}$, 由于 $\sigma d$ 量 反映了界面缺陷大小的量级, 因而说明在我 们的超晶格样品中其界面处的缺陷大小并 不因绝缘层 PrBCO 层的增厚而增加, 另外 $c$ 轴的大小为 $1.17 \mathrm{~nm}$ 左右, 比通常的纯 $\mathrm{YBCO}$ 膜的 $c$ 轴要短, 但我们没有发现象 Fullerton 等所观察到的 $c$ 轴随绝缘层 PrBCO 层的增 厚而降低的现象，模拟计算时我们均假设了层厚的微小波动量为 $5 \%$ 左右,这在所有样品中几 乎没有多大变化.

表 1 计算模拟时所用到的 $\mathrm{YBCO}$ 薄膜 $\mathrm{Cu}-\mathrm{O}$ 面间距的值及相应的有关修正参数

\begin{tabular}{cccccccc}
\hline 样品 & $\mathrm{Cu}_{1}-\mathrm{Ba} / \mathrm{nm}$ & $\mathrm{Ba}-\mathrm{Cu}_{2} / \mathrm{nm}$ & $\mathrm{Cu}_{2}-\mathrm{Y} / \mathrm{nm}$ & $\mathrm{c}$-axis $/ \mathrm{nm}$ & $\sigma_{d} / \mathrm{nm}$ & 扩散量 $/ \% \cdot \sigma_{n} \cdot n^{-1} / \%$ \\
\hline $\mathrm{YP147}(1: 1)$ & 0.217 & 0.199 & 0.169 & 1.170 & 0.01 & 5 & 5 \\
$\mathrm{YP148(1:3)}$ & 0.217 & 0.198 & 0.170 & 1.170 & 0.003 & 5 & 5 \\
$\mathrm{YP149(1:5)}$ & 0.217 & 0.203 & 0.167 & 1.174 & 0.010 & 10 & 5 \\
$\mathrm{YP164}(1: 8)$ & 0.217 & 0.204 & 0.166 & 1.174 & 0.002 & 5 & 5 \\
$\mathrm{YP155(2:15)}$ & 0.217 & 0.198 & 0.172 & 1.172 & 0.003 & 15 & 5 \\
\hline
\end{tabular}

总之,由上我们可以清楚地看出尽管我们所制备得的超晶格样品具有很好的 $c$ 外延取向, 但仍明显地存在着精细结构中的不完整性, 如缺陷, 层厚波动等, 虽然我们没有找到这种结构 上的不完整性与 $\mathrm{PrBCO}$ 厚度的直接关系, 但我们认为在解释 $T_{\mathrm{c}}$ 随 $\mathrm{PrBCO}$ 层的增厚而下降 (如图 5 所示, 由 $\mathrm{AC}$ - 磁化率测量得到, $T_{\mathrm{c}}$ 相当于 $\gamma^{\prime}$ 的 onset 点的温度值) 时, 不应该忽略这种 结构上的不完整性. 


\section{3 结论}

对单层和双层 $\mathrm{YBCO}$ 原胞层厚的 $\mathrm{YBCO} / \mathrm{PrBCO}$ 超晶格的 $\mathrm{X}$ 射线衍射分别作了实验测 量和计算模拟, 比较两者谱图发现尽管样品具有很好的 $c$ 轴取向, 但仍然存在着明显的缺陷, 诸如界面台阶无序、 $Y$ 原子与 $\operatorname{Pr}$ 原子地少量扩散及界面地不平整性等, 并且我们认为这种结 构上的不完整性可能对解释超晶格样品的 $T_{\mathrm{c}}$ 随着绝缘层 PrBCO 层厚的增加而下降的机制起 一定的帮助作用.

\section{参考文献}

1 Triscone $\mathrm{J} \mathrm{M}$, Fischer $\mathrm{O}$, Bruner $\mathrm{O}$ et al. $\mathrm{YBa}_{2} \mathrm{Cu}_{3} \mathrm{O}_{7} / \mathrm{PrBa}_{2} \mathrm{Cu}_{3} \mathrm{O}$, superlattices: properties of ultrathin superconducting layers seperated by insulating layers. Phys Rev Lett, 1990, 64: 804; Lowndes D H, Norton D P, Budai $\mathrm{J}$ D. Superconductivity in nosymmetric epitaxial $\mathrm{YBa}_{2} \mathrm{Cu}_{3} \mathrm{O}_{7-x} / \mathrm{PrBa}_{2} \mathrm{Cu}_{3} \mathrm{O}_{7-x}$ superlattices: the superconducting behavior of Cu-O bilayers. Phys Rev Lett, 1990, 65: 1160

2 Jakob G, Przyslupski P. High-superconducting critical current density in $\mathrm{YBa}_{2} \mathrm{Cu}_{3} \mathrm{O}_{7} / \mathrm{PrBa}_{2} \mathrm{Cu}_{3} \mathrm{O}_{7}$ superlattics. Appl Phys Lett, 1991, 159: 1626

3 Wood R F. Spin polarons, hold filling and high- $T_{c}$ superonductivity in $\mathrm{YBCO}$ and $\mathrm{PrBCO}$ alloys and layered films. Phys Rev Lett, 1991, 66:829

4 Rasolt M, Edis T, Tesanovic Z. Kosterlitz-Thouless transition and charge redistribution in the superconductivity of YBCO/PBCO superlattices. Phys Rev Lett, 1991, 66: 2927

5 Wu J Z, Ting C S, Chu W K et al. Proximity effect on the superonducting transition temeprature of $\mathrm{YBa}_{2} \mathrm{Cu}_{3} \mathrm{O}_{7-x} /$ $\mathrm{PrBa}_{2} \mathrm{Cu}_{3} \mathrm{O}_{7-x}$ superlattiœes. Phys Rev, B, 1991, 44:411

6 Jakob G. Hahn Th. Transport properties of $\mathrm{YBa}_{2} \mathrm{Cu}_{3} \mathrm{O}_{7} / \mathrm{PrBa}_{2} \mathrm{CuO}_{7}$ superlattiœes. Euro Phys Lett, 1992, 19: 135

7 Schuller I K. New class of layered materials. Phs Rev Lett, 1980, 44:1 597; Fullerton E E, Guimpel J, Nakamura O et al. Structure of high- $T_{\mathrm{c}}$ superlattices. Phys Rev Lett, 1992, 69:2859 